\title{
Taxonomical Evaluation of Plant Chloroplastic Markers by Bayesian Classifier
}

\author{
Luisa Matiz-Ceron ${ }^{1,2}$, Alejandro Reyes ${ }^{1,2 *}$ and Juan Anzola ${ }^{1,2,3 *}$ \\ ${ }^{1}$ Research Group in Computational Biology and Microbial Ecology, Department of Biological Sciences, Universidad de los \\ Andes, Bogotá, Colombia, ${ }^{2}$ Max Planck Tandem Group in Computational Biology, Universidad de los Andes, Bogotá, \\ Colombia, ${ }^{3}$ Department of Engineering and Natural Sciences, Universidad Central, Bogotá, Colombia
}

\section{OPEN ACCESS}

Edited by:

Itay Mayrose,

Tel Aviv University, Israel

Reviewed by:

Panagiotis Madesis,

University of Thessaly, Greece

Shing Hei Zhan,

Fusion Genomics Corporation,

Canada

*Correspondence:

Alejandro Reyes

a.reyes@uniandes.edu.co

Juan Anzola

juan.anzola@corpogen.org

Specialty section:

This article was submitted to Plant Systematics and Evolution,

a section of the journal

Frontiers in Plant Science

Received: 24 September 2021 Accepted: 29 December 2021 Published: 03 February 2022

Citation:

Matiz-Ceron L, Reyes A and Anzola J (2022) Taxonomical Evaluation of Plant Chloroplastic Markers by Bayesian Classifier.

Front. Plant Sci. 12:782663. doi: $10.3389 /$ fpls.2021.782663
DNA barcodes are standardized sequences that range between 400 and $800 \mathrm{bp}$, vary at different taxonomic levels, and make it possible to assign sequences to species that have been previously taxonomically characterized. Several DNA barcodes have been postulated for plants, nonetheless, their classification potential has not been evaluated for metabarcoding, and as a result, it would appear as none of them excels above the others in this area. One tool that has been widely used and served as a baseline when evaluating new approaches is Naiive Bayesian Classifiers (NBC). The present study aims at evaluating the classification power of several plant chloroplast genetic markers that have been proposed as barcodes (trnL, rpoB, rbcL, matK, psbA-trnH, and $p s b K)$ using an NBC. We performed the classification at different taxonomic levels, and identified problematic genera when resolution was desired. We propose matK and trnL as potential candidate markers with resolution up to genus level. Some problematic genera within certain families could lead to the misclassification no matter which marker is used (i.e., Aegilops, Gueldenstaedtia, Helianthus, Oryza, Shorea, Thysananthus, and Triticum). Finally, we suggest recommendations for the taxonomic identification of plants in samples with potential mixtures.

Keywords: Naïve Bayesian classifier, metabarcoding, matK, $t r n L$, taxonomic classification, chloroplast

\section{INTRODUCTION}

In recent years, DNA barcoding has been proposed as a method to survey biodiversity in the field (Hebert et al., 2003; Gross, 2012). DNA barcodes were proposed originally for animal classification (Hebert et al., 2003), but later, they were proposed for plants as well (Kress and Erickson, 2007). DNA barcoding represents an efficient tool for the identification of cryptic or invasive species (Lopez-Vaamonde et al., 2021), conservation, and community ecology (Hollingsworth et al., 2011; Yessoufou et al., 2013; Bezeng et al., 2017). This tool is based on the conserved DNA biomarkers with more interspecific than intraspecific variability creating a barcoding gap (Čandek and Kuntner, 2015), which allows the possibility to identify an organism at different taxonomic levels. An appropriate marker must have the following characteristics: (a) a significant genetic diversity, based on the desired resolution of the barcode, with conserved flanking sites to enable primer design; (b) An appropriate size for DNA extraction and amplification protocols; and (c) to be as generalist as possible, it should be present in all the targeted taxons (Kress et al., 2005; CBOL Plant Working Group, 2009). In recent years, advances in sequencing technologies have opened the possibility of ecological surveys based on sequencing data. Examples of these are the $16 S$ rRNA gene in prokaryotes, the Internal Transcribed Spacer (ITS) region in fungi and the Cytochrome Oxidase I 
(COI) in animals. Despite these advances, there are several taxonomic groups for which no ideal marker has been found for classification purposes or metabarcoding analysis.

Given the low mutation rate of mitochondrial DNA, mitochondrial cytochrome oxidase I (COI) cannot be used in plants (CBOL Plant Working Group, 2009; Li et al., 2021). In consequence, extensive search within nuclear and chloroplast genomes have been performed to identify suitable regions for barcode design. Three main regions have been proposed, the ribulose-1,5-bisphosphate carboxylase/oxygenase large subunit or RuBisCO large subunit ( $r b c L)$, maturase K (matK), and Internal Transcribed Spacer 2 (ITS2) (Cowan et al., 2006; CBOL Plant Working Group, 2009; China Plant BOL Group, 2011). However, none of them have the precision that COI displays for animals (Pang et al., 2012), they do not have sufficient resolution for groups, such as lichens, bryophytes, or ferns (Kress and Erickson, 2007), and are ineffective for samples with degraded or fragmented DNA (Mallott et al., 2018). In consequence, other regions, such as Transfer RNA T-L spacer (trnL), photosystem II protein D1-Transfer RNA H (psbA-trnH), and Photosystem II $K$ protein-I spacer ( $p s b K-I$ ) have been proposed (Lahaye et al., 2008; Ghorbani et al., 2017; Mallott et al., 2018; Thakur et al., 2019). Similarly, the presence of disruptions due to differences in the demography of species, or rare but recorded events in which different species share the same haplotype, generate the need for new strategies, such as the combination of markers and the evaluation of different regions (Xiao-Xian and Zhe-Kun, 2007; CBOL Plant Working Group, 2009; Pang et al., 2012; Wang et al., 2017; Mallott et al., 2018).

Since the majority of these markers (matK, $r b c L, t r n L$, $p s b A$ trnH, $p s b K-I$, and $r p o B$ ) have been studied in specific plant families, their potential for general taxonomic classification is still unknown, or their capacity to discriminate individual species within a complex mixture (metabarcoding), for example, when processing fecal or soil samples (Lahaye et al., 2008; Gillespie et al., 2009; Seberg and Petersen, 2009; Nicolalde-Morejón et al., 2010; De Groot et al., 2011; Korotkova et al., 2011; Diekmann et al., 2012; Gere et al., 2013; Lee et al., 2017). Therefore, emerging strategies combining markers, such as matK + rbcL could represent a better approximation for some plant groups species; nevertheless, the evaluation of genes distantly located, or that requires multiple amplicons, are not suitable for metabarcoding analysis since it is currently impossible to link both amplicons to a given origin or DNA molecule.

For any given marker, as important as the intrinsic genetic variability is the availability of a tool that will detect it and be able to assign it accurately and precisely to a taxonomical level. One such tool that is widely used for pattern recognition in DNA sequences and serves as a standard when evaluating new classification approaches is the Naïve Bayesian Classifier (NBC) (Busia et al., 2019). NBC is a machine learning technique that generates a supervised classification model based on a training set. Given that NBC assumes that the input variables are independent and have an equal effect on the classification outcome, each variable (or parameter) must be learned by the classifier from the training set, allowing it to form a posterior probability of assignment or classification. This simple model allows for the evaluation of huge datasets. Moreover, the effectiveness of this classifier has been demonstrated in applications, such as text classification, medical diagnostics, and applications for data administration (Domingos and Pazzani, 1997; Hellerstein et al., 2000). Databases, such as the Ribosomal Database Project (RDP), and software, such as QIIME2 and MOTHUR use Bayesian approaches for the taxonomical classification of nucleic acid sequences (Wang et al., 2007; Schloss et al., 2009; Cole et al., 2013 Bokulich et al., 2018; Bolyen et al., 2018).

The use of Bayesian Classifiers in taxonomic classification requires a reference set of DNA sequences with their respective taxonomic labels. Furthermore, sequence classification depends on the type of marker, the training set, and length of the k-mer (Werner et al., 2012). Here, we used six chloroplast gene sequences to evaluate their classification power (matK, $r b c L$, trnL, rpoB, psbA-trnH, and psbK-I) using an NBC, and to analyze their performance when considering variables, such as marker selection, and representativity in databases. We provided a statistical evaluation of the different marker performance based on the distribution of sensitivity and accuracy (F1 score). Finally, we evaluated genera with low classification performance with the aim to find biological explanations for their misclassification and make some suggestions for researchers who suspect that they have species of these genera in their samples.

\section{MATERIALS AND METHODS}

\section{Data Preparation}

Six chloroplastic markers (trnL, rpoB, rbcL, matK, psbA$\operatorname{trnH}$, and $p s b K)$ were chosen based on the representation in public databases and frequent use in literature. Sequences were downloaded from GenBank on September of 2018 using as entrez query: txid3193 (corresponding to embryophyta), with minimum length of 50 bp (e.g., txid3193[Organism:exp] AND tRNL[Gene Name] AND 50:400000000[Sequence Length] NOT UNVERIFIED [Title]). The same query was used for each of the other markers (matK, rbcL, rpoB, psbA$\operatorname{trn} H$, and $p s b K-I)$ by replacing the corresponding gene name. Sequences were downloaded in GenBank format and imported into Geneious R9 (Biomatters, New Zealand). Features (genes) were extracted in FASTA format using the "extract annotations" feature of Geneious. The taxonomic distribution of each marker is represented in Table 1. The accession numbers of the six chloroplast markers are available in Supplementary Table 1 .

\section{Extraction of Taxonomic Information From Gene Markers}

The accession number of each sequence was used to download the corresponding taxonomic information from the National Center for Biotechnology Information (NCBI) taxonomy. Taxonomic information per sequence was organized into the corresponding taxonomic levels: Phylum (P), Class (C), Order (O), Family (F), Genus (G), and Species (S). This was done using inhouse scripts wrapped around the software ETE (Huerta-Cepas et al., 2016). The taxonomic information was assigned to every sequence following the pattern of the Greengenes database for 
bacterial classification (DeSantis et al., 2006). The sequences went through two independent filters: first, maintaining only those that had complete taxonomic classification, as reported in NCBI Taxonomy DB; second, keeping those with almost full-length for the corresponding marker. Sequence number variation after every filter is available in Supplementary Table 2. Size filtering was performed in Geneious.

Finally, we balanced the dataset to reduce possible biases in the data (over- or under-representation of certain species). Each DNA marker was balanced according to the number of sequences present at the species level. Two different datasets were generated for each molecular marker: the first one with species having a minimum of two sequences and a maximum of 20 sequences per species (the dataset 2-20), and the second one having a minimum of five sequences and a maximum of 20 sequences per species (the dataset 5-20). For both cases, species that had more than 20 sequences were randomly subsampled to 20 sequences. On one hand, the 5-20 dataset was generated to evaluate the performance of every marker at different taxonomic levels. On the other hand, the 2-20 dataset was only used to determine how sensible does the model is for underrepresented taxons (i.e., species with only 2-4 sequences available). The script used to perform this balancing is available in https:/osf.io/qtz59/?view_ only=538ab7719073498abfaea0ab1b29d2ba.

\section{Classification Algorithm}

We used the NBC as implemented in MOTHUR (Schloss et al., 2009). This implementation follows the algorithm described by Wang and collaborators (Wang et al., 2007). Here we used the script classify.seqs from MOTHUR which requires two input files, one with the sequences to build the classification model and another with the full taxonomy for each input sequence separated by each taxonomic level. As output, it will generate a classification with the name of the sequence and a full taxonomic classification with the bootstrap value obtained at each level.

To the best of our knowledge, the algorithm, as described by Wang and collaborators, take each sequence from the dataset, and decompose them into a vector of words of certain size $k(\mathrm{k}-$ mers), size 8 by default, generating a vector of $k$-mers and their corresponding abundances. Those vectors are used to calculate a joint probability and hence a probability of assignment for any new sequence (Wang et al., 2007). Confidence estimation of the assignment is implemented in MOTHUR as well. For this, for every sequence that is going to be classified, one hundred (100) random subsets of $1 / 8$ of the k-mer set are chosen randomly and the resulting vector is used for classification. The confidence estimation is then the number of times (out of 100) or bootstrap that the given assignment is obtained. Traditionally an $80 \%$ bootstrap value has been selected as having a high precision and accuracy and hence, it was selected as a threshold for subsequent analyses and only results with classifications above that threshold were considered as "trusted" assignments.

As a cross-validation methodology, we chose the "leaveone-out" method (Wang et al., 2007), as it is one of the most exhaustive cross-validation methods. This method is implemented in MOTHUR in the script classify.seqs. Briefly, one sequence is extracted from the dataset, the remaining sequences are used to train the Bayesian model and then the extracted sequence is classified against the recently trained model. This procedure was repeated for each sequence in the dataset and for all markers. The resulting classification for each of the "left out" sequences were then used to calculate the accuracy and precision of the models. The scripts used to run MOTHUR and the final trained model generated are available here https://osf.io/qtz59/ ?view_only=538ab7719073498abfaea0ab1b29d2ba.

\section{Statistical and Graphical Analysis}

The program RStudio version 1.1.456 (RStudio Team, 2015) was used for graph generation and statistical analysis. The caret package (Kuhn, 2008) was used for the calculation of F1 score. The F1 metric was chosen because it represents the balance between correct and incorrect classifications (precision and recall). Precision is defined as the number of True Positives divided by the number of True Positives plus the number of False Positives. Recall is the number of True Positives divided by the number of True Positives plus the number of False Negatives. F1 score $=$ Precision/Recall. A model that performs perfectly would have an F1 score equal to 1, whereas a model that performs poorly would have a score toward zero (0). An important aspect to highlight the use of F1 score is that it ignores the true negatives which in this type of classifiers are usually the large majority and would bias the values obtained. A confusion matrix was constructed using the real and predicted taxonomic assignments for the 5-20 dataset. All data and calculations are available in Supplementary Table 3. The F1 score for a given genus was calculated as the average value for all the species within the genus. This information was represented on a heatmap. We selected genera with the lowest classification scores-F1 $(<0.25)$ - to explore possible reasons that may be responsible for the constitutive misclassification of the sequences. A multiple sequence alignment (MSA) was generated using MUSCLE V3.81 (Edgar, 2004). The graph was elaborated using the graphic tool for alignment evaluation AliView (Larsson, 2014). The R-package ggplot2 was used for the graphical representation of F1 score (Wickham, 2016).

\section{RESULTS}

\section{Dataset Exploration}

In this study, we used two different datasets (2-20) and (5-20) to determine marker performance and dataset bias, respectively, for plant classification in metabarcoding. The taxonomic distribution is shown in Table 1. Sequence and species count for both 2-20 and 5-20 datasets are presented in Table 2, showing the highest number of genera and species for markers $\operatorname{trn} L, r b c L$, and matK. Moreover, $r b c L$ was the marker with the highest number of sequences and species available in databases after quality control and filtering (Table 2, 2-20 dataset). Species with just one sequence as representative is impossible to be used for training a model. It is important to notice the high decrease in the number of species due to their representation by one single sequence, about $50 \%$ of all the dataset for most markers was lost in the filtering process (Supplementary Table 2). 
TABLE 1 | Taxonomical representation of selected markers at different taxonomical levels.

\begin{tabular}{lcccccc}
\hline Barcodes & Species & Genus & Family & Order & Class & Phylum \\
\hline rbcL & 30,208 & 8,151 & 670 & 136 & 17 & 1 \\
matK & 26,382 & 6,377 & 483 & 113 & 13 & 1 \\
trnL & 22,027 & 5,023 & 451 & 116 & 13 & 1 \\
psbA-trnH & 5,059 & 1,102 & 204 & 78 & 12 & 1 \\
rpoB & 3,996 & 1,736 & 305 & 94 & 12 & 1 \\
psbK & 3,579 & 1,465 & 249 & 88 & 11 & 1 \\
\hline $\begin{array}{l}\text { The number } \\
\text { marker gene is shown. }\end{array}$ & & & & & &
\end{tabular}

TABLE 2 | Number of species and sequences in the datasets used in this study.

\begin{tabular}{lccccc}
\hline \multirow{2}{*}{ Barcodes } & \multicolumn{2}{c}{$\mathbf{2 - 2 0}$} & & \multicolumn{2}{c}{$\mathbf{5 - 2 0}$} \\
\cline { 2 - 3 } \cline { 5 - 6 } & \# of species & \# of sequences & \# of species & \# of sequences \\
\hline rbcL & 9,119 & 27,836 & & 1,125 & 8,405 \\
matK & 7,732 & 24,413 & & 946 & 7,998 \\
trnL & 5,218 & 19,355 & & 919 & 8,861 \\
psbA-trnH & 1,744 & 6,838 & & 370 & 3,400 \\
rpoB & 2,721 & 6,667 & & 102 & 872 \\
psbK & 2,694 & 6,589 & & 99 & 850 \\
\hline
\end{tabular}

\section{Distribution of Bootstrap Values}

Classification with the NBC was used as implemented in MOTHUR and described in methods, for both datasets at all taxonomic levels. At higher taxonomic levels, classification was more accurate, with a decrease in accuracy at lower levels, such as genus and species. At the genus level, most of the assigned sequences exhibit bootstraps values with over $90 \%$ value, regardless of the marker used, whereas for species, confidence of the classification decreased, with values in the (60-69) and (70-79) range, with $\operatorname{trnL}$ as a good example (Figure 1). However, the largest category corresponded to those sequences assigned with $>95 \%$ bootstrap with both datasets (Figure 1 and Supplementary Figure 1). Here, we used a cut-off bootstrap value of $80 \%$ for evaluation of the taxonomical assignments.

\section{Marker Performance and Dataset Bias}

We tested the accuracy at different taxonomic levels. The average accuracy of phylum, class, order, and family for every marker, for both datasets (the 5-20 and 2-20), was above $98 \%$ of correctly assigned sequences (Figures 2A,B). This implies a similar classification power for all the markers at these taxonomic levels. It is worth highlighting the fact that our final dataset contained only one phylum, thus, classification at this taxonomic level could be biased due to the absence of other phylum. At the genus and species level, a sharp decline in correct assignments was observed, with genus at about $90 \%$ and species at about $80 \%$ of correctly assigned sequences for the 5-20 dataset (Figure 2A). There was a significant difference in the performance between the 5-20 vs. 2-20 datasets, with the former displaying a better performance at genus and species levels (95 and 80\%, respectively; Figure 2A), than the latter (85 and 70\%, respectively; Figure $2 \mathrm{~B}$ ) verifying the impact of underrepresented taxons in the classification power of the algorithm. In consequence, we selected the 5-20 dataset for subsequent analyses.

We evaluated the performance of each marker at the genus and species level (Figure 3). We observe that matK and $\operatorname{trnL}$ are the markers with the highest rate of correct assignments at the genus level. For species, mat $K$ and $r p o B$ seems to be the best markers (fewer incorrect assignments); however, at this level none of the markers surpasses $95 \%$ assignment accuracy (the number of sequences per marker is represented in Table 2).

\section{Using Classifiers on Problematic Genera}

In general, fewer than $15 \%$ of the sequences were misclassified at the species level. Furthermore, those misclassified sequences tend to belong to a reduced number of genera. Hence, we decided to identify the genera with the lowest classification performance at the species level to determine potential reasons for their problematic classification. We used the F1 score for the selection of those problematic genera. For a correct interpretation of the F1 score, it is necessary to remember that the closer the score is to 1 , the higher the quality of the prediction generated by the precision value (proportion of sequences correctly classified or true positives) and the recall (proportion of sequences belonging to one category and classified as another or false positives). The respective cumulative percentage of genus classification for every marker based on the F1 score is shown in Figure 4 and Supplementary Table 3. Essentially, we found three different scenarios:

(a) The genus has one or multiple markers and at least one performs well: these genera ( $n=684$ or $65 \%)$ have an F1 score greater than 0.25 in one or multiple markers. However, there are some cases $(n=34$ or $3.21 \%$ ) where the genera have an F1 score below 0.25 for at least one marker but were able to be classified correctly by at least one another marker.

(b) The genus has only one marker and it performs poorly. These genera ( $n=36$ or $3.4 \%$ ) have only data (sequences) available for one marker and the F1 score $\leq 0.25$. In this case, there was no further information to evaluate these genera.

(c) The genus has multiple markers and none of them performs well: There are genera ( $n=7$ or $0.66 \%)$ with multiple markers and with F1 score $<0.25$ in all of them constituting an important dataset for further analysis into the possible causes for the misclassification. We call these genera "problematic genera."

Among the selected problematic genera, seven showed consistent problematic assignments (Aegilops, Gueldenstaedtia, Helianthus, Oryza, Shorea, Thysananthus, and Triticum), and no marker was efficient in classifying these taxa. The heatmap in Figure 5 shows an example of the widespread classification problems in these genera for matK (the other markers are in Supplementary Figure 2). Most of the misclassification problems occur between species originating from the same genus; however, some misclassifications are seen at the genus level for the family Poaceae between Aegilops and Triticum (Figure 5). This happens for markers $p s b K, r b c L$, and $r p o B$. Finally, we performed an analysis of problematic genera and their respective species for every marker by a Multiple Sequence Alignment to evaluate if the low performance of the assignment task was correlated 


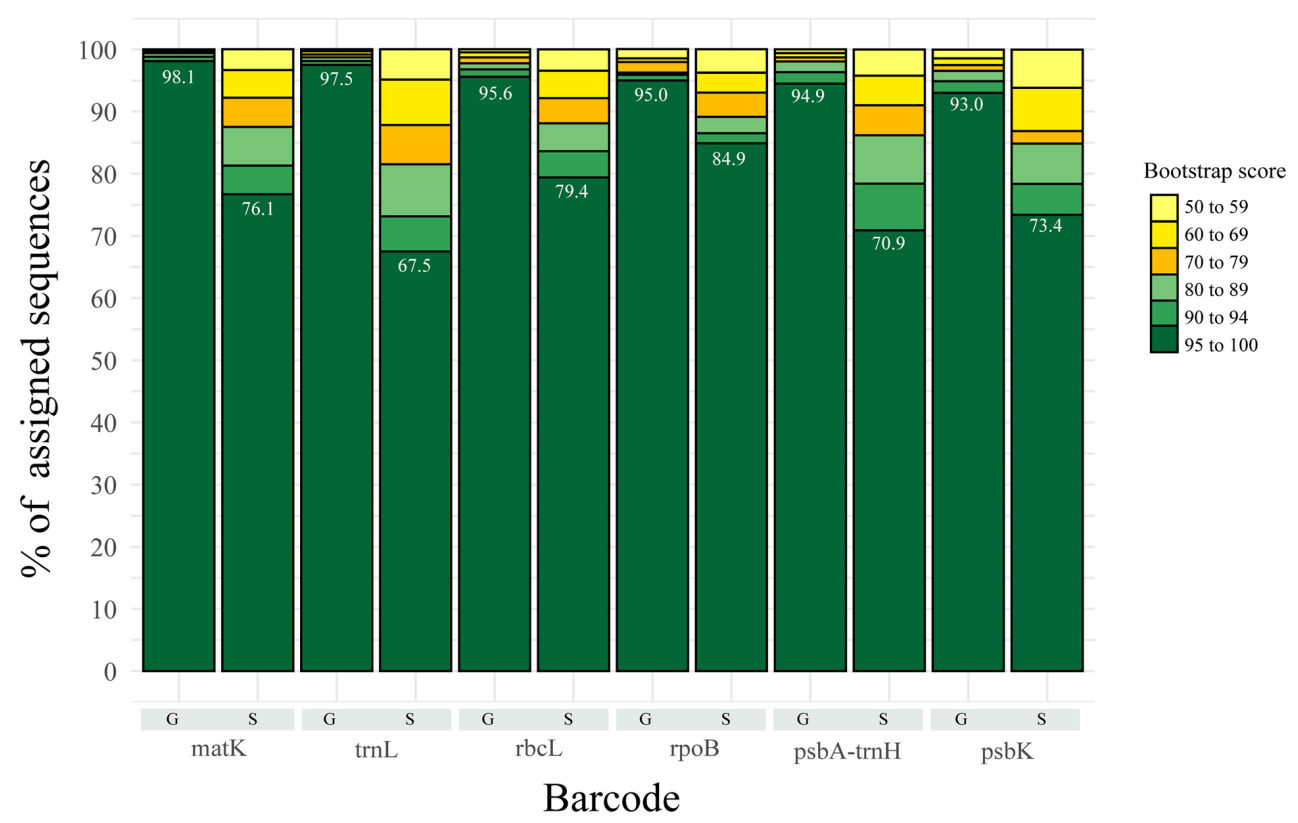

FIGURE 1 | Representation of the percentage of assigned sequences (i.e., classified) by the Bayesian Classifier at species and genus level for the 5-20 datasets. Shades of green represent the fraction of sequences with bootstrap values greater than 80 (proposed threshold for accuracy). Yellow represents the fraction of sequences with bootstrap values lower than 80 , thus not used for further analysis.

with low intraspecific variability. Figure $\mathbf{6}$ is an example of the genus Shorea for the marker trnL. As expected, we found very low variation in the multiple alignment within these problematic genera.

\section{DISCUSSION}

The current study shows an evaluation of the most relevant markers used in literature for plant classification, using one of the methods that has demonstrated greater effectiveness of classification in metabarcoding and with the maximum number of sequences that can be obtained in public databases. This process was performed to identify the markers with the highest accuracy at different taxonomic levels for metabarcoding analysis and to identify genera with problematic species. Not surprisingly, $r b c L$ was the marker with the highest number of sequences, given that historically it has been the most used marker (Kress and Erickson, 2007; Hollingsworth et al., 2011).

\section{Dataset Exploration}

Our comparative results between the 5-20 and 2-20 datasets show that better performance is achieved with datasets with good representation per class (5-20 dataset), rather than with datasets with a higher number of classes, but lower representation per class. This is true for the most classification methods. It further highlights the importance of increasing the reports of sequences from different taxonomic lineages in public databases, since only a good representation of a given lineage will allow a proper model training for accurate classification.

\section{Markers Examination by Bayesian Classifiers}

Bayesian classifiers have been successfully used in metabarcoding strategies. The method employed here has the added benefit of bootstrapping the k-mers used to reduce the chance of overfitting and guarantee that no single k-mer is responsible for a given classification. Thus, classifications with high bootstrap values show the robustness of the method. In our case, we needed to find a balance between high confidence in the assignment, given by the bootstrap value, and the high percentage of classification. We selected a frequently used $80 \%$ bootstrap threshold for our analyses, which allowed the classification of over $80 \%$ of the sequences at species level and over $95 \%$ of the sequences at the genus level or above. At species level, mat $K$ and $r p o B$ appear to be the best markers (fewer incorrect assignments); however, at this level, none of the markers surpasses $95 \%$ of assignment accuracy. This level of accuracy, which is acceptable in other fields of machine learning, is probably too low to be considered a "good classifier" at the species level. According to this result, it is recommended to use markers above the $98 \%$ accuracy at the genus level, which are matK and trnL.

When analyzing the performance of the individual markers, we want to highlight mat $K$ and its performance on taxonomic assignment, even with datasets of sparse representation, such as the 2-20 dataset. Its outstanding performance could be attributed to the high interspecific variability of the marker (Mankga et al., 2013; Jiménez-Mejías et al., 2016; Elansary et al., 2017). Our results were consistent with the recommendations from other authors based on the low performance of other single markers at the species level 


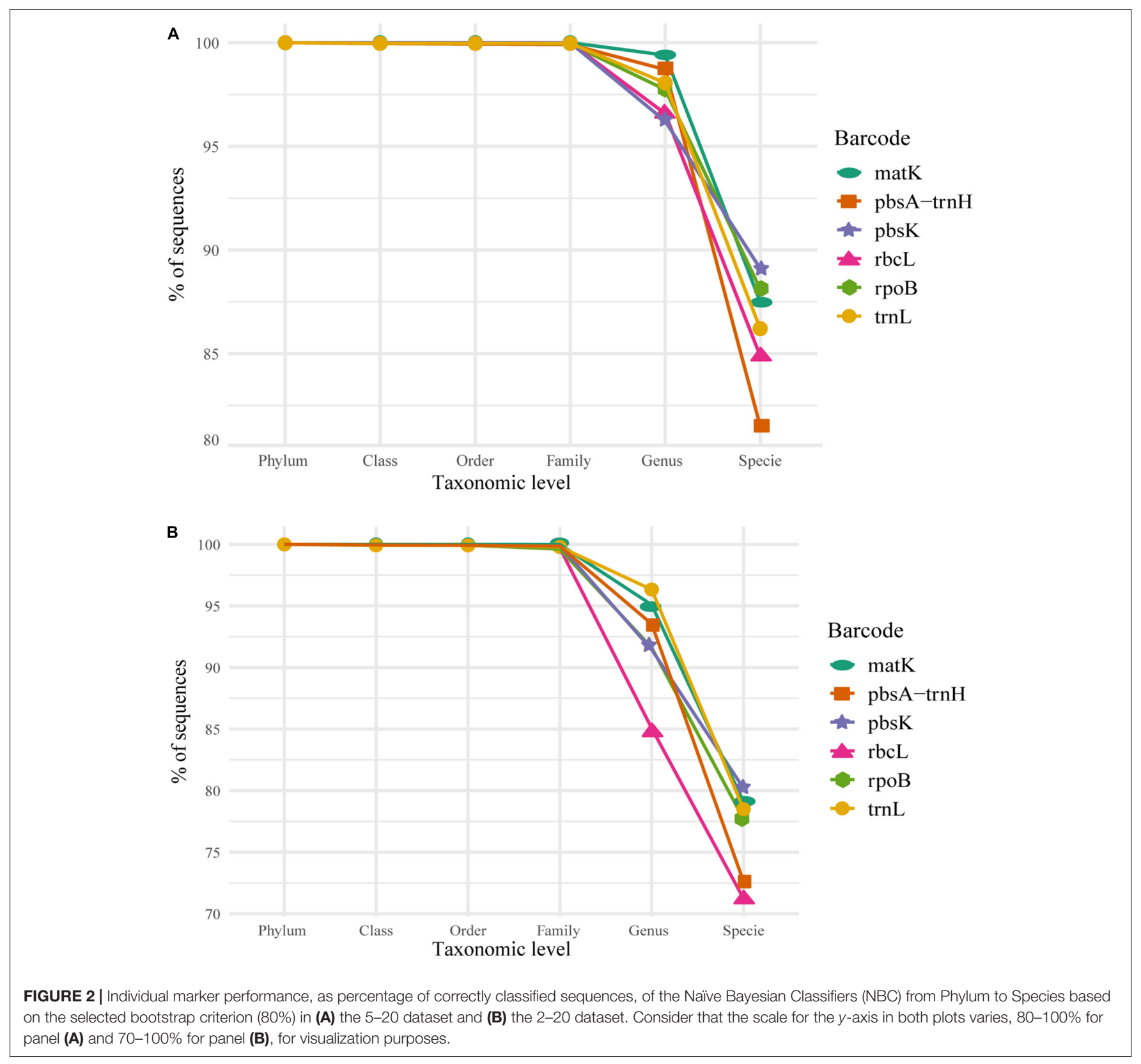

(Whittall et al., 2010; China Plant BOL Group, 2011; De Groot et al., 2011; Hollingsworth et al., 2011; Gere et al., 2013; Chen et al., 2016; Kress, 2017; Menezes et al., 2018). Although a proposed alternative for barcoding was the combination of multiple single gene markers, this approach is unsuitable in the context of metabarcoding given that it is currently technically impossible to tie two or more markers to an individual in an environmental sample, unless the markers were next to each other and amplifiable as a single amplicon. Our results indicate that for single marker classification, trnL and matK are the best choices when classifying up to genus level and are resilient to low sequence representation in databases. In addition, we recommend matK, which was the marker with the best performance overall at species level. However, further studies are necessary to determine flanking regions of matK that could improve the species classification. For general plant classification, we agree with the recommendation from several authors of using $m a t K$ in combination with other genes (Braukmann et al., 2017; Xu et al., 2018; Li et al., 2021).

\section{Problematic Genera}

For the genera with problematic classifications, we found three types of behavior (Supplementary Table 3). First, we identified some genera (3.2\%) with very low F1 score with one specific marker, but with enough resolution for other markers (e.g., Citrus, Adenophora, Oenothera, Rosa, and Vitis), thus representing a limitation of a given marker-genus pair for classification. Second, there were some genera $(3.4 \%)$ with 


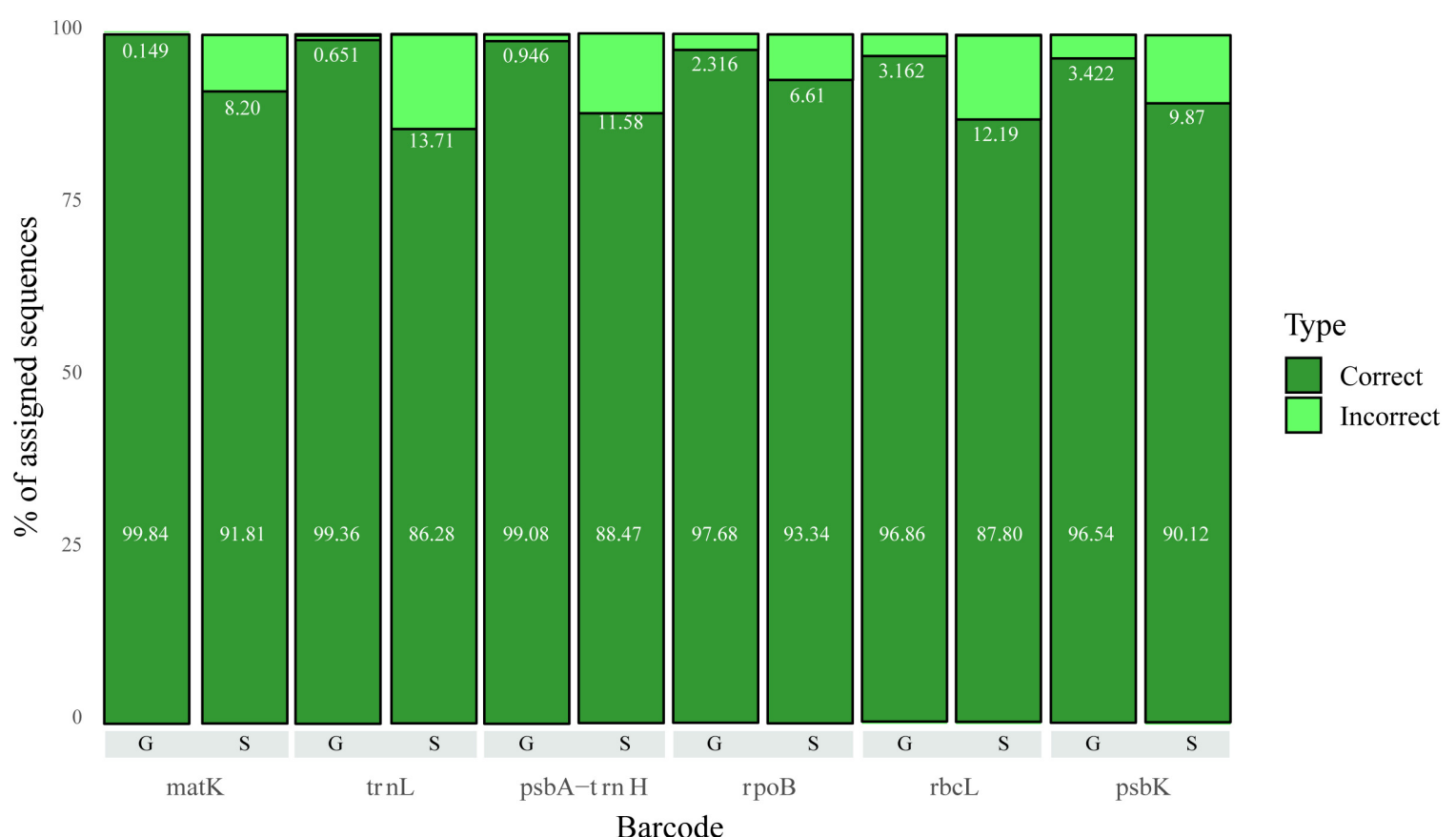

FIGURE 3 | Correct and Incorrect classifications for Genus (G) and Species (S) levels for all markers evaluated in the 5-20 dataset. The value corresponding to the percentage of correct assignments is shown in the bottom of the bar, while the value for incorrect assignments is right below the corresponding light green bar.

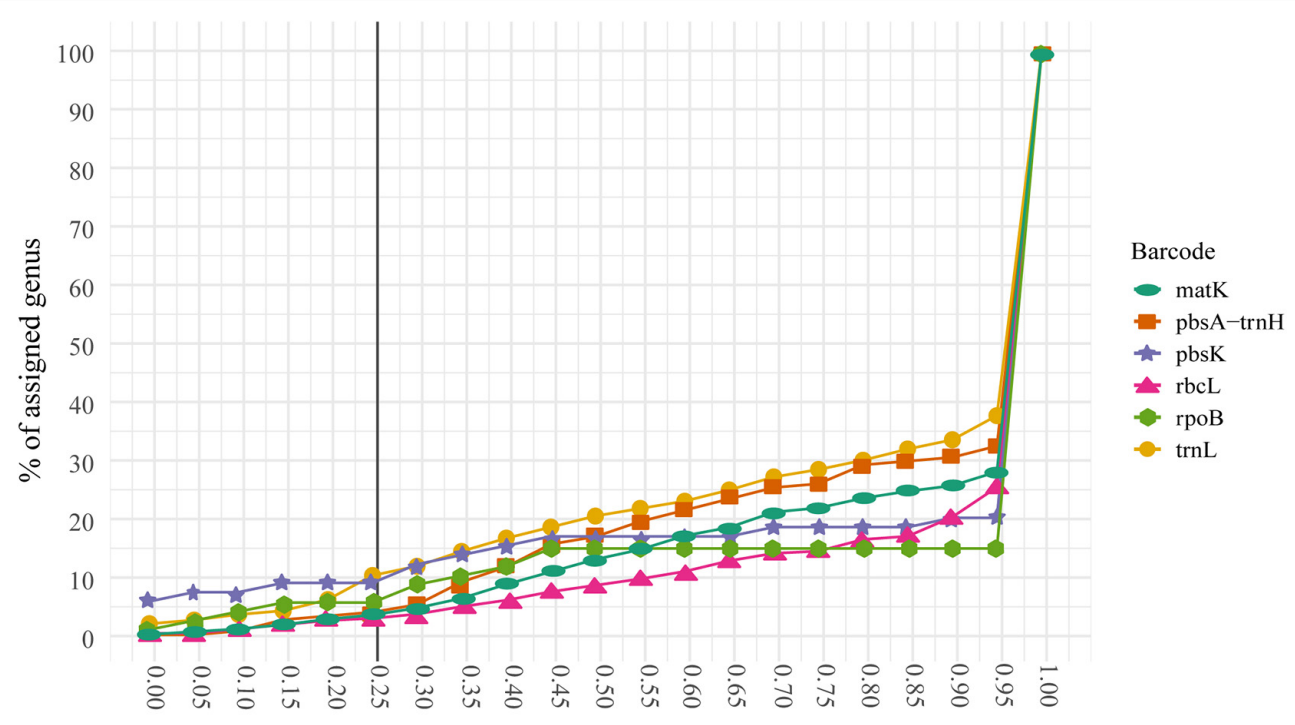

Accumulative F1 score

FIGURE 4 | Cumulative F1 score for the 5-20 dataset for every marker. The cumulative percentage of assigned genus at every F1 score threshold is shown. Thus, $100 \%$ of the genera are assigned at a cut-off of 1.0 or below. Notice that less than $10 \%$ of the genera had an F1 score of 0.25 or below and $65 \%$ of the genera shows a score of 0.9 or above for every marker. The shown line defines the threshold for the genera that will be handpicked to evaluate their possible causes of misclassification.

information for only one marker, making it impossible to compare or gather more information from those, highlighting the importance of generating more data on those markers for under-represented taxa. Moreover, most of these genera had a low number of sequences which itself implies an associated factor to their low classification accuracy. Third and finally, there was a set of genera $(0.66 \%)$ with two or more markers generating misclassifications. For this final case, we found a total of seven genera Aegilops (Poaceae, goatgrasses), Gueldenstaedtia (Fabaceae, legume), Helianthus (Asteraceae, sunflower), Oryza (Poaceae, rice), Shorea (Dipterocarpaceae, lauan), Thysananthus (Lejeuneaceae, liverwort), and Triticum (Poaceae, wheat). 

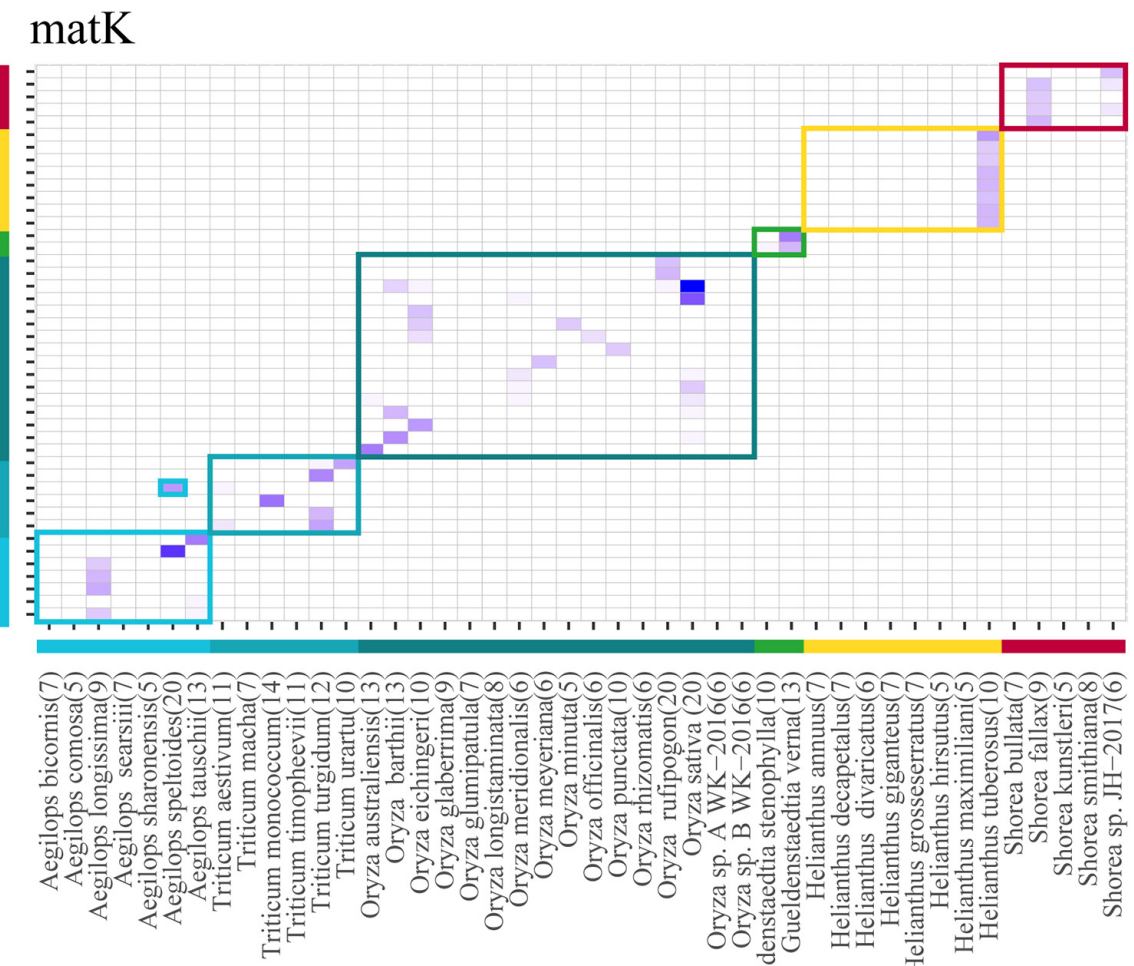

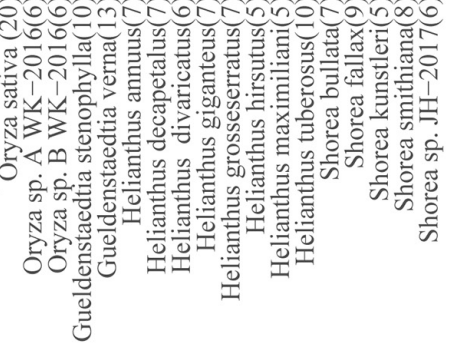

\section{Color by Family \\ Poaceae \\ Fabaceae \\ Asteraceae \\ Dipterocarpaceae}

FIGURE 5 | Heatmap corresponding to the confusion matrix of assignment for problematic genera in the case of the matK marker. Species from selected genera are depicted in the same order in the $x$ - and $y$-axis. Horizontal band color and vertical bar colors at the bottom and side of the heatmap correspond to the families evaluated. Squares enclose their corresponding species. Species in $X$-axis (original) are predicted as one of the $Y$-axis (predicted). The numbers within parenthesis represents the number of sequences for that species.

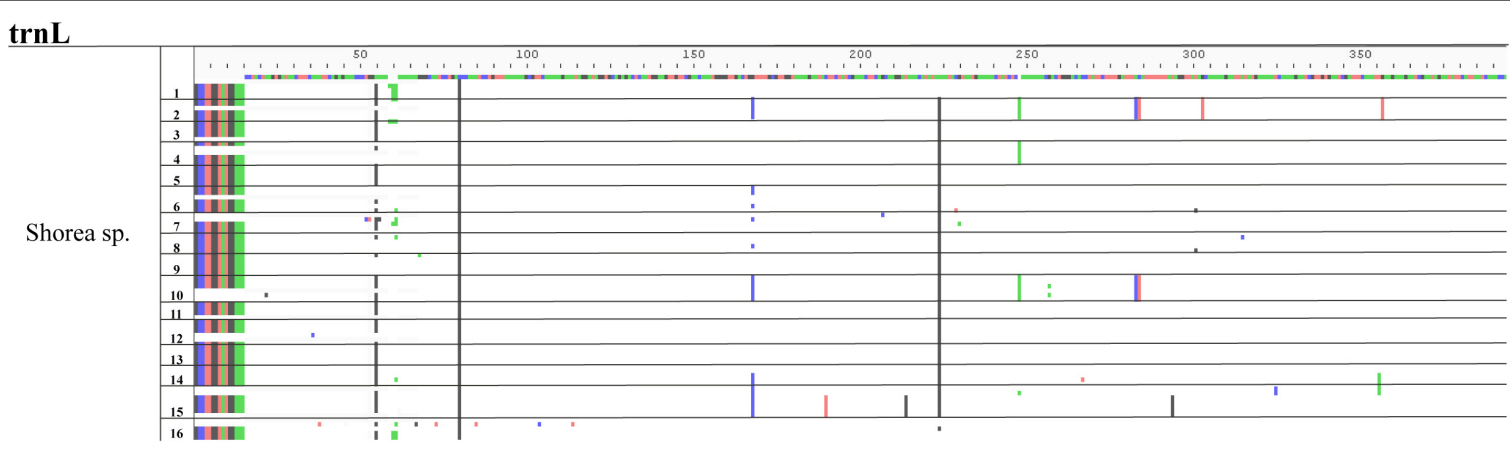

FIGURE 6 | Multiple sequences alignment (MSA) example of species variation from the genus Shorea using trnL marker. The sequence on the top is the alignment consensus of the genus Shorea sp. The different colors below this line indicate the variations within the sequences. The numbers at one side represents the species of this genus. Horizontal lines represent divisions between the different species. Notice that there are no specific variations that could work as specific markers for any given species.

A deeper look at these genera allowed important observations. Gueldenstaedtia is a small genus of Fabaceae that is very similar to the Tibetia genus (Xie et al., 2016). Most of the misclassified species in Gueldenstaedtia were assigned as Tibetia species. We found a similar situation with the genera Shorea and Thysananthus. Shorea is a very important genus related to timber and wood products. Tsumura et al. (2011) reported that some species within Shorea have identical sequences for multiple chloroplast regions, indicating that it may be difficult to discriminate between closely related species. In their manuscript, they propose a method for the identification of species of this genus and suggest using other non-chloroplast-based markers, such as ITS for the identification of Shorea. Finally, for Thysananthus, one of the largest genera of liverworts that has been monographed worldwide, some authors suggest that given their morphological overlap, the molecular evidence and the lack of morphological characters separating them from Mastigolejeunea, they should be merged 
as a single genus (Sukkharak and Gradstein, 2017). Thus, the misclassification on those three genera seems to be more related to the lack of biological divergence than the performance of the markers themselves.

The genera Aegilops and Helianthus showed a pattern that regardless of the species being assigned, all assignments were collapsed to a single species. For Aegilops, this pattern was observed in many of the evaluated markers, such as matK (Figure 5, notice most species are classified as Aegilops longissima). For Helianthus, the pattern was presented in matK, $r b c L, p s b K$, and $r p o B$ (Supplementary Figure 2). A second pattern of apparent random assignments was found for Oryza, Triticum, Citrus, and Shorea, just to mention some of the examples. This behavior was found for more than one marker ( $m a t K, r p o B, r b c L, p s b K$, and $p s b A-t r n H$ ). Analyzing those genera and their respective species using MSA, we noticed a very low or non-existent variation among the sequences (Figure 6) pointing again at very low biological divergence among the species of the genus, and likely the cause of the low F1 score for those genera.

We identified a particular pattern among Aegilops and Triticum, both belonging to the Poaceae family. In these genera, for different markers, occurs a classification of Aegilops species as Triticum ones, and vice versa. In the most recent phylogenetic classification of Poaceae, these two genera have been assigned as being part of the subtribe Triticinae (Soreng et al., 2015) based on methods of maximum likelihood on matK and $n d h F$ markers. In those genera, there is evidence of hybridization (Loureiro et al., 2009; Tsunewaki, 2009; Zhang et al., 2010) using allopolyploidization as the major force leading to the diversification during the evolution of Triticum species. Aegilops has been characterized as a wild relative of Triticum (wheat). Cultivated wheats and their close wild relatives belong to the genus Triticum, a member of the tribe Triticeae, which contains 300 species (Clayton and Renvoize, 1986). Together, this evidence suggests some of the possible causes of classification problems with the assignment of these two genera.

Hollingsworth et al. (2011) suggests seven key factors that may lead to a lower level of success in species discrimination, such as hybridization, polyploidy, life history, breeding systems, species history, level of taxonomic "splitting," and seed dispersal. In all our problematic genera (Aegilops, Gueldenstaedtia, Helianthus, Oryza, Shorea, Thysananthus, and Triticum) those patterns were present. In Aegilops, there is evidence of allopolyploid, containing multiple chloroplast haplotypes, each identical to haplotypes of the diploid progenitor species, indicating multiple origins as the major source of variation (Meimberg et al., 2009). In Triticum, there was evidence of hybridization and polyploidy. Hybridization occurs between wheat cultivars because mixed cultivation of different wheats with different ploidies is a tradition and still common practice in the Middle East and Transcaucasia. Furthermore, wild wheat species can be involved in hybrid swarms in regions where they naturally grow in and around the areas of wheat cultivation (Matsuoka, 2011). There was a similar pattern with the genus Oryza and Helianthus given their human domestication and their economic importance as food source (Blackman et al., 2011; Molina et al., 2011; Kantar et al., 2012; Civáň et al., 2015; Badouin et al., 2017; Stein et al., 2018).

\section{Caveats and Recommendations}

Finally, it is important to highlight that the taxonomy source of the evaluated sequences was GenBank. This is a database of primary sequences where the submitter gives the taxonomic assignment of the uploaded sequence and thus, it is prone to human error. A certain error percentage is expected by using such a database. We tried to minimize the error by using several representative sequences per species, but this was limited in some cases.

Our results show that the NBC is a tool that could be used for plant classification. Based on the results of this classifier for the taxonomic levels: class, order, or family, any of the evaluated markers would sufficiently fulfill the expected accuracy and precision. For classification at the genus level, trnL and matK are the recommended choice due to their high performance of classification, even on taxons with low number of sequenced representatives (2-5 sequences per taxon). We only evaluated classifications based on Naïve Bayesian models; however, other methods using machine learning models, such as support-vector machine (SVM) could be of interest for future validations. Due to the limitations in metabarcoding studies and with long-read sequencing technologies becoming more prevalent, we propose the evaluation of chloroplast regions that contains more than one gene, identical to the regions close to $\operatorname{trn} L$, matK, $p s b A$-trnH, $r b c L$, and $r p o B$ using NBC as a modeling method.

Some specific combinations of marker-genus were problematic for classification; however, several of them could be assigned with other markers. We further analyzed the possible reasons of multiple marker misclassification for the genera Aegilops, Gueldenstaedtia, Helianthus, Oryza, Shorea, Thysananthus, and Triticum, identifying in all cases that it was likely related to biological conditions, such as hybridization, polyploidy, and evolutionary history, and not due to the algorithm or technical difficulties. Barcodes are powerful tools for sequence classification and plants are no exception. However, only a thorough analysis, such as the one performed in the current study can provide evidence of the usability of the different markers and their limitations. Here, we used the most common molecular markers together with all the available sequences on public databases and a state-of-the-art classification method to determine the best performing marker for each taxon on potential interest and, finally, release a Green Genes-like database to be used by the researchers on their own research.

\section{DATA AVAILABILITY STATEMENT}

The original contributions presented in the study are included in the article/Supplementary Material, further inquiries can be directed to the corresponding authors.

\section{AUTHOR CONTRIBUTIONS}

AR and JA contributed to the conception, designed of the study, and reviewed the manuscript. JA organized the database. LM-C performed the statistical and classification analysis, 
wrote the draft, and final version of the manuscript. All authors contributed to manuscript revision, read, and approved the submitted version.

\section{ACKNOWLEDGMENTS}

We thank the School of Sciences at Universidad de los Andes seed project number INV-2019-85-1788 for their support for LM-C to present the project at an International Conference. We would also like to thank the Max Planck Tandem Group and Universidad de los Andes for funding tuition and stipend for LM-C. A preprint of this document could found in Authorea

\section{REFERENCES}

Badouin, H., Gouzy, J., Grassa, C. J., Murat, F., Staton, S. E., Cottret, L., et al. (2017). The sunflower genome provides insights into oil metabolism, flowering and Asterid evolution. Nature 546:148. doi: 10.1038/nature22380

Bezeng, B. S., Davies, T. J., Daru, B. H., Kabongo, R. M., Maurin, O., Yessoufou, K., et al. (2017). Ten years of barcoding at the African Centre for DNA barcoding. Genome 60, 629-638. doi: 10.1139/gen-2016-0198

Blackman, B. K., Rasmussen, D. A., Strasburg, J. L., Raduski, A. R., Burke, J. M., Knapp, S. J., et al. (2011). Contributions of flowering time genes to sunflower domestication and improvement. Genetics 187, 271-287. doi: 10.1534/genetics. 110.121327

Bokulich, N. A., Kaehler, B. D., Rideout, J. R., Dillon, M., Bolyen, E., Knight, R., et al. (2018). Optimizing taxonomic classification of marker-gene amplicon sequences with QIIME 2's q2-feature-classifier plugin. Microbiome 6:90. doi: 10.1186/s40168-018-0470- z

Bolyen, E., Rideout, J. R., Dillon, M. R., Bokulich, N. A., Abnet, C., Al-Ghalith, G. A., et al. (2018). QIIME 2: reproducible, interactive, scalable, and extensible microbiome data science (No. e27295v1). PeerJ 6:e27295v2. doi: 10.7287/peerj. preprints.27295v2

Braukmann, T. W., Kuzmina, M. L., Sills, J., Zakharov, E. V., and Hebert, P. D. (2017). Testing the efficacy of DNA barcodes for identifying the vascular plants of Canada. PLoS One 12:e0169515. doi: 10.1371/journal.pone.0169515

Busia, A., Dahl, G. E., Fannjiang, C., Alexander, D. H., Dorfman, E., Poplin, R., et al. (2019). A deep learning approach to pattern recognition for short DNA sequences. bioRxiv [Preprint]. 353474 doi: 10.1093/bib/bbaa049

Čandek, K., and Kuntner, M. (2015). DNA barcoding gap: reliable species identification over morphological and geographical scales. Mol. Ecol. Resour. 15, 268-277. doi: 10.1111/1755-0998.12304

CBOL Plant Working Group (2009). A DNA barcode for land plants. Proc. Natl. Acad. Sci. U.S.A. 106, 12794-12797.

Chen, Z., Feng, K., Grover, C. E., Li, P., Liu, F., Wang, Y., et al. (2016). Chloroplast DNA structural variation, phylogeny, and age of divergence among diploid cotton species. PLoS One 11:e0157183. doi: 10.1371/journal.pone.0157183

China Plant BOL Group (2011). Comparative analysis of a large dataset indicates that internal transcribed spacer (ITS) should be incorporated into the core barcode for seed plants. Proc. Natl. Acad. Sci. U.S.A. 108, 19641-19646. doi: 10.1073/pnas.1104551108

Civáň, P., Craig, H., Cox, C. J., and Brown, T. A. (2015). Three geographically separate domestications of Asian rice. Nat. Plants 1:15164. doi: 10.1038/nplants. 2015.164

Clayton, W. D., and Renvoize, S. A. (1986). Genera graminum: Grasses of the World, Vol. 13. London: Her Majesty's Stationery.

Cole, J. R., Wang, Q., Fish, J. A., Chai, B., McGarrell, D. M., Sun, Y., et al. (2013). Ribosomal database project: data and tools for high throughput rRNA analysis. Nucleic Acids Res. 42, D633-D642. doi: 10.1093/nar/gkt1244

Cowan, R. S., Chase, M. W., Kress, W. J., and Savolainen, V. (2006). 300,000 species to identify: problems, progress, and prospects in DNA barcoding of land plants. Taxon 55, 611-616. doi: 10.2307/25065638

De Groot, G. A., During, H. J., Maas, J. W., Schneider, H., Vogel, J. C., and Erkens, R. H. (2011). Use of rbcL and trnL-F as a two-locus DNA barcode and as a thesis document at Universidad de los Andes repository. Special thanks to the IT Services Department and ExaCore-IT Core-facility of the Vice Presidency for Research and Creation at the Universidad de Los Andes for high-performance computing services and technical assistance.

\section{SUPPLEMENTARY MATERIAL}

The Supplementary Material for this article can be found online at: https://www.frontiersin.org/articles/10.3389/fpls.2021. 782663/full\#supplementary-material

for identification of NW-European ferns: an ecological perspective. PLoS One 6:e16371. doi: 10.1371/journal.pone.0016371

DeSantis, T. Z., Hugenholtz, P., Larsen, N., Rojas, M., Brodie, E. L., Keller, K., et al. (2006). Greengenes, a chimera-checked 16S rRNA gene database and workbench compatible with ARB. Appl. Environ. Microbiol. 72, 5069-5072. doi: 10.1128/AEM.03006-05

Diekmann, K., Hodkinson, T. R., and Barth, S. (2012). New chloroplast microsatellite markers suitable for assessing genetic diversity of Lolium perenne and other related grass species. Annl. Bot. 110, 1327-1339. doi: 10.1093/aob/ mcs044

Domingos, P., and Pazzani, M. (1997). On the optimality of the simple Bayesian classifier under zero-one loss. Mach. Learn. 29, 103-130.

Edgar, R. C. (2004). MUSCLE: multiple sequence alignment with high accuracy and high throughput. Nucleic Acids Res. 32, 1792-1797. doi: 10.1093/nar/gkh340

Elansary, H., Ashfaq, M., Ali, H. M., and Yessoufou, K. (2017). The first initiative of DNA barcoding of ornamental plants from Egypt and potential applications in horticulture industry. PLoS One 12:e0172170. doi: 10.1371/journal.pone. 0172170

Gere, J., Yessoufou, K., Daru, B. H., Mankga, L. T., Maurin, O., and van der Bank, M. (2013). Incorporating trnH-psbA to the core DNA barcodes improves significantly species discrimination within southern African Combretaceae. ZooKeys 365:129. doi: 10.3897/zookeys.365.5728

Ghorbani, A., Saeedi, Y., and de Boer, H. J. (2017). Unidentifiable by morphology: DNA barcoding of plant material in local markets in Iran. PLoS One 12:e0175722. doi: 10.1371/journal.pone.0175722

Gillespie, L. J., Soreng, R. J., and Jacobs, S. W. (2009). Phylogenetic relationships of Australian Poa (Poaceae: Poinae), including molecular evidence for two new genera, Saxipoa and Sylvipoa. Aust. Syst. Bot. 22, 413-436.

Gross, M. (2012). Barcoding biodiversity. Curr. Biol. 22, R73-R76.

Hebert, P. D., Cywinska, A., Ball, S. L., and DeWaard, J. R. (2003). Biological identifications through DNA barcodes. Proc. R. Soc. Lond. B Biol. Sci. 270, 313-321. doi: 10.1098/rspb.2002.2218

Hellerstein, J. L., Jayram, T. S., and Rish, I. (2000). Recognizing End-User Transactions In Performance Management. Hawthorne, NY: IBM Thomas J. Watson Research Division.

Hollingsworth, P. M., Graham, S. W., and Little, D. P. (2011). Choosing and using a plant DNA barcode. PLoS One 6:e19254. doi: 10.1371/journal.pone.0019254

Huerta-Cepas, J., Serra, F., and Bork, P. (2016). ETE 3: reconstruction, analysis and visualization of phylogenomic data. Mol. Biol. Evol. 33, 1635-1638. doi: 10.1093/molbev/msw046

Jiménez-Mejías, P., Hahn, M., Lueders, K., Starr, J. R., Brown, B. H., Chouinard, B. N., et al. (2016). Megaphylogenetic specimen-level approaches to the Carex (Cyperaceae) phylogeny using ITS, ETS, and matK sequences: implications for classification. Syst. Bot. 41, 500-518.

Kantar, M., Betts, K., Hulke, B. S., Stupar, R. M., and Wyse, D. (2012). Breaking tuber dormancy in Helianthus tuberosus L. and interspecific hybrids of Helianthus annuus L. $\times$ Helianthus tuberosus. Hortscience 47, 1342-1346. doi: 10.21273/hortsci.47.9.1342

Korotkova, N., Borsch, T., Quandt, D., Taylor, N. P., Müller, K. F., and Barthlott, W. (2011). What does it take to resolve relationships and to identify species with 
molecular markers? An example from the epiphytic Rhipsalideae (Cactaceae). Am. J. Bot. 98, 1549-1572. doi: 10.3732/ajb.1000502

Kress, W. J. (2017). Plant DNA barcodes: applications today and in the future. J. Syst. Evol. 55, 291-307. doi: 10.1111/jse.12254

Kress, W. J., and Erickson, D. L. (2007). A two-locus global DNA barcode for land plants: the coding rbcL gene complements the non-coding trnH-psbA apacer region. PLoS One 2:e508. doi: 10.1371/journal.pone.0000508

Kress, W. J., Wurdack, K. J., Zimmer, E. A., Weigt, L. A., and Janzen, D. H. (2005). Use of DNA barcodes to identify flowering plants. Proc. Natl. Acad. Sci. U.S.A. 102, 8369-8374. doi: 10.1073/pnas.0503123102

Kuhn, M. (2008). Building predictive models in R using the caret package. J. Stat. Softw. 28, 1-26.

Lahaye, R. R., Savolainen, V., Duthoit, S., Maurin, O., and Van der Bank, M. (2008). A test of Psbk-Psbi And Atpf-Atph As potential plant dna barcodes using the flora of the kruger national park (South Africa) as a model system. Nat. Precedings 3:1.

Larsson, A. (2014). AliView: a fast and lightweight alignment viewer and editor for large data sets. Bioinformatics 30, 3276-3278. doi: 10.1093/bioinformatics/ btu531

Lee, S. C., Wang, C. H., Yen, C. E., and Chang, C. (2017). DNA barcode and identification of the varieties and provenances of Taiwan's domestic and imported made teas using ribosomal internal transcribed spacer 2 sequences. J. Food Drug Anal. 25, 260-274. doi: 10.1016/j.jfda.2016.06.008

Li, H., Xiao, W., Tong, T., Li, Y., Zhang, M., Lin, X., et al. (2021). The specific DNA barcodes based on chloroplast genes for species identification of Orchidaceae plants. Sci. Rep. 11, 1-15. doi: 10.1038/s41598-021-81087-w

Lopez-Vaamonde, C., Kirichenko, N., Cama, A., Doorenweerd, C., Godfray, H. C. J., Guiguet, A., et al. (2021). Evaluating DNA barcoding for species identification and discovery in European gracillariid moths. Front. Ecol. Evol. 9:66. doi: 10.3389/fevo.2021.626752

Loureiro, I., Escorial, C., García-Baudin, J. M., and Chueca, C. (2009). Hybridization, fertility and herbicide resistance of hybrids between wheat and Aegilops biuncialis. Agron. Sustain. Dev. 29, 237-245.

Mallott, E. K., Garber, P. A., and Malhi, R. S. (2018). TrnL outperforms rbcL as a DNA metabarcoding marker when compared with the observed plant component of the diet of wild white-faced capuchins (Cebus capucinus. Primates). PLoS One 13:e0199556. doi: 10.1371/journal.pone.019 9556

Mankga, L. T., Yessoufou, K., Moteetee, A. M., Daru, B. H., and van der Bank, M. (2013). Efficacy of the core DNA barcodes in identifying processed and poorly conserved plant materials commonly used in South African traditional medicine. Zookeys 365:215. doi: 10.3897/zookeys.365.5730

Matsuoka, Y. (2011). Evolution of polyploid Triticum wheats under cultivation: the role of domestication, natural hybridization, and allopolyploid speciation in their diversification. Plant Cell Physiol. 52, 750-764. doi: 10.1093/pcp/pcr018

Meimberg, H., Rice, K. J., Milan, N. F., Njoku, C. C., and McKay, J. K. (2009). Multiple origins promote the ecological amplitude of allopolyploid Aegilops (Poaceae). Am. J. Bot. 96, 1262-1273. doi: 10.3732/ajb.0800345

Menezes, A. P., Resende-Moreira, L. C., Buzatti, R. S., Nazareno, A. G., Carlsen, M., Lobo, F. P., et al. (2018). Chloroplast genomes of Byrsonima species (Malpighiaceae): comparative analysis and screening of high divergence sequences. Sci. Rep. 8:2210. doi: 10.1038/s41598-018-20189-4

Molina, J., Sikora, M., Garud, N., Flowers, J. M., Rubinstein, S., Reynolds, A., et al. (2011). Molecular evidence for a single evolutionary origin of domesticated rice. Proc.Natl. Acad. Sci. 108, 8351-8356. doi: 10.1073/pnas.1104686108

Nicolalde-Morejón, F., Vergara-Silva, F., González-Astorga, J., and Stevenson, D. W. (2010). Character-based, population-level DNA barcoding in Mexican species of Zamia L.(Zamiaceae: Cycadales). Mitochondrial DNA 21, 51-59. doi: 10.3109/19401736.2010.539215

Pang, X., Liu, C., Shi, L., Liu, R., Liang, D., Li, H., et al. (2012). Utility of the trnHpsbA intergenic spacer region and its combinations as plant DNA barcodes: a meta-analysis. PLoS One 7:e48833. doi: 10.1371/journal.pone.0048833

RStudio Team (2015). RStudio: Integrated Development for R. RStudio, Inc. Boston, MA: RStudio.

Schloss, P. D., Westcott, S. L., Ryabin, T., Hall, J. R., Hartmann, M., Hollister, E. B., et al. (2009). Introducing MOTHUR: open-source, platform-independent, community-supported software for describing and comparing microbial communities. Appl. Environ. Microbiol. 75, 7537-7541. doi: 10.1128/AEM. 01541-09
Seberg, O., and Petersen, G. (2009). How many loci does it take to DNA barcode a crocus? PLoS One 4:e4598. doi: 10.1371/journal.pone.0004598

Soreng, R. J., Peterson, P. M., Romaschenko, K., Davidse, G., Zuloaga, F. O., Judziewicz, E. J., et al. (2015). A worldwide phylogenetic classification of the Poaceae (Gramineae). J. Syst. Evol. 53, 117-137. doi: 10.1360/aps06148

Stein, J. C., Yu, Y., Copetti, D., Zwickl, D. J., Zhang, L., Zhang, C., et al. (2018). Genomes of 13 domesticated and wild rice relatives highlight genetic conservation, turnover and innovation across the genus Oryza. Nat. Genet. 50, 285-296.

Sukkharak, P., and Gradstein, S. R. (2017). Phylogenetic study of Mastigolejeunea (Marchantiophyta: Lejeuneaceae) and an amended circumscription of the genus Thysananthus. Phytotaxa 326, 91-107. doi: 10.11646/phytotaxa.326.2.1

Thakur, V. V., Tiwari, S., Tripathi, N., and Tiwari, G. (2019). Molecular identification of medicinal plants with amplicon length polymorphism using universal DNA barcodes of the atpF-atpH, trnL and trnH-psbA regions. 3. Biotech 9, 1-10. doi: 10.1007/s13205-019-1724-6

Tsumura, Y., Kado, T., Yoshida, K., Abe, H., Ohtani, M., Taguchi, Y., et al. (2011). Molecular database for classifying Shorea species (Dipterocarpaceae) and techniques for checking the legitimacy of timber and wood products. J. Plant Res. 124, 35-48. doi: 10.1007/s10265-010-0348-z

Tsunewaki, K. (2009). Plasmon analysis in the Triticum-Aegilops complex. Breed. Sci. 59, 455-470.

Wang, A., Gopurenko, D., Wu, H., and Lepschi, B. (2017). Evaluation of six candidate DNA barcode loci for identification of five important invasive grasses in eastern Australia. PLoS One 12:e0175338. doi: 10.1371/journal.pone.0175338

Wang, Q., Garrity, G. M., Tiedje, J. M., and Cole, J. R. (2007). Naïve Bayesian classifier for rapid assignment of rRNA sequences into the new bacterial taxonomy. Appl. Environ. Microbiol. 73, 5261-5267. doi: 10.1128/AEM.0006207

Werner, J. J., Koren, O., Hugenholtz, P., DeSantis, T. Z., Walters, W. A., Caporaso, J. G., et al. (2012). Impact of training sets on classification of high-throughput bacterial 16s rRNA gene surveys. ISME J. 6, 94-103. doi: 10.1038/ismej.2011.82

Whittall, J. B., Syring, J., Parks, M., Buenrostro, J., Dick, C., Liston, A., et al. (2010). Finding a (pine) needle in a haystack: chloroplast genome sequence divergence in rare and widespread pines. Mol. Ecol. 19, 100-114. doi: 10.1111/j.1365-294X. 2009.04474.x

Wickham, H. (2016). ggplot2: Elegant Graphics For Data Analysis. Berlin: Springer. Xiao-Xian, L., and Zhe-Kun, Z. (2007). The higher-level phylogeny of monocots based On MatK, rbcL and $18 \mathrm{~S}$ r. Zhi Wu Fen Lei Xue Bao 45, 113-133.

Xie, Y. P., Meng, Y., Sun, H., and Nie, Z. L. (2016). Molecular phylogeny of Gueldenstaedtia and Tibetia (Fabaceae) and their biogeographic differentiation within Eastern Asia. PLoS One 11:e0162982. doi: 10.1371/journal.pone.0162982

Xu, S. Z., Li, Z. Y., and Jin, X. H. (2018). DNA barcoding of invasive plants in China: a resource for identifying invasive plants. Mol. Ecol. Resour. 18, 128-136. doi: 10.1111/1755-0998.12715

Yessoufou, K., Davies, T. J., Maurin, O., Kuzmina, M., Schaefer, H., van der Bank, M., et al. (2013). Large herbivores favour species diversity but have mixed impacts on phylogenetic community structure in an A frican savanna ecosystem. J. Ecol. 101, 614-625. doi: 10.1111/1365-2745.12059

Zhang, L. Q., Liu, D. C., Zheng, Y. L., Yan, Z. H., Dai, S. F., Li, Y. F., et al. (2010). Frequent occurrence of unreduced gametes in Triticum turgidum-Aegilops tauschii hybrids. Euphytica 172, 285-294.

Conflict of Interest: The authors declare that the research was conducted in the absence of any commercial or financial relationships that could be construed as a potential conflict of interest.

Publisher's Note: All claims expressed in this article are solely those of the authors and do not necessarily represent those of their affiliated organizations, or those of the publisher, the editors and the reviewers. Any product that may be evaluated in this article, or claim that may be made by its manufacturer, is not guaranteed or endorsed by the publisher.

Copyright (c) 2022 Matiz-Ceron, Reyes and Anzola. This is an open-access article distributed under the terms of the Creative Commons Attribution License (CC BY). The use, distribution or reproduction in other forums is permitted, provided the original author(s) and the copyright owner(s) are credited and that the original publication in this journal is cited, in accordance with accepted academic practice. No use, distribution or reproduction is permitted which does not comply with these terms. 